\title{
Glass transition of semi-crystalline PLLA with different morphologies as studied by dynamic mechanical analysis
}

\author{
Ricardo Picciochi • Yaming Wang • Natália M. Alves • \\ João F. Mano
}

Received: 19 June 2006 / Accepted: 25 September 2006 / Published online: 28 November 2006

(C) Springer-Verlag 2006

\begin{abstract}
Poly(L-lactic acid) was crystallized from the glassy state at different temperatures to produce fully transformed semi-crystalline specimens exhibiting different lamellar morphologies. The materials were tested by dynamic mechanical analysis, where a $T_{\mathrm{g}}$ decrease was found with an increasing crystallization temperature. Considering a three-phase model, this tendency was related to the corresponding increase in the thickness of the rigid amorphous phase. It is suggested that this phase could, in some extent, accommodate through local translational/ rotational motions the cooperative motions taking place within the mobile amorphous phase. This could be due to the non-compact structure of the cooperatively rearranging regions, which can present a string-like or fractal structure in their edges. The width of the loss factor peak associated to the glass transition increases with increasing crystallization temperature, suggesting an increase in the broadness of the distribution of relaxation times. The drop in the storage modulus across $T_{\mathrm{g}}$ varies systematically with the crystallization temperature in the different materials and could be correlated with the crystalline content. Above $T_{\mathrm{g}}$, the loss factor exhibits a plateau-like behaviour at significantly high values, which seems to be a rather general behaviour in semi-crystalline systems that could be related to the contribution of pure irreversible flow in the overall viscoelastic behaviour.
\end{abstract}

\footnotetext{
R. Picciochi $\cdot$ Y. Wang $\cdot$ N. M. Alves $\cdot$ J. F. Mano

3B's Research Group-Biomaterials,

Biodegradables and Biomimetics, University of Minho,

4710-057 Braga, Portugal

R. Picciochi $\cdot$ Y. Wang $\cdot$ N. M. Alves $\cdot$ J. F. Mano $(\bowtie)$

Polymer Engineering Department, University of Minho,

Campus of Azurém,

4800-058 Guimarães, Portugal

e-mail: jmano@dep.uminho.pt
}

Keywords DMA - Confined glass transition .

Semi-crystalline polymers · Glass transition dynamics

\section{Introduction}

Polylactides are widely well-known biodegradable and biocompatible systems used both in implantable medical devices and in environmental applications [1-3]. The glass transition of poly(L-lactic acid) (PLLA) is not far above room or physiological temperature, and thus, this phenomenon should be well studied as it could influence the general behaviour of the material under use. For example, it was shown that structural relaxation, studied at $37{ }^{\circ} \mathrm{C}$ by differential scanning calorimetry (DSC) and microhardness, could highly influence the mechanical performance of amorphous PLLA along the time [4]. The features of the amorphous phase strongly depend on the existence of crystallinity: Upon crystallization, the glass transition temperature $\left(T_{\mathrm{g}}\right)$ increases, and the distribution of relaxation times enlarges [5-7]. PLLA is known to have a slow crystallization rate (see for example [8] for TMDSC studies), and it is therefore possible to obtain materials with a wide range of crystalline contents. For intermediate crystallinity degrees, two distinct glass transitions could be observed $[6,7,9]$. The one with the lower temperature exhibits similar features of the glass transition dynamics for the amorphous materials, whereas the one with the higher temperature should be assigned to the motions within the inter-spherulitic amorphous phase constrained by the existence of the crystalline phase. These two processes, for PLLA, are not correlated, and their relative contributions are highly dependent on the degree of crystalline transformation [9]. The study of the higher temperature glass transition process is especially interesting, as its 
behaviour will be dependent upon the characteristics of the nanometric confinement provoked by the stiff wall of the lamellae. It is well known that a fraction of the amorphous phase in fully transformed semi-crystalline polymers does not contribute to the glass transition of the mobile amorphous phase nor to the heat of fusion. The wellestablished three-phase model considers the existence of a non-oriented nanophase between the mobile amorphous phase and the crystalline lamellae (see [10] and references cited therein). The scheme in Fig. 1 shows the arrangement of the three phases, where their thicknesses can be obtained from small-angle X-ray scattering combined with DSC data.

The degree of geometrical confinement felt by the mobile amorphous phase between the crystalline lamellae will depend on the corresponding layer thickness $L_{\mathrm{ma}}$. However, it would be interesting to understand if the rigid amorphous layer could influence independently the glass transition features. In a previous work, some DSC data, combined with both wide- and small-angle X-ray scattering, demonstrate that PLLA crystallized at different temperatures could be an adequate model to investigate the influence of the rigid amorphous phase in the segmental mobility of the mobile amorphous regions [11]. In the present work, further discussion in this context will be provided using dynamic mechanical analysis (DMA). Such results can also be useful to understand the general solid-state rheological properties of PLLA samples fully crystallized at different temperatures. In this context, the viscoelastic properties around $T_{\mathrm{g}}$ were previously extensively discussed for a semi-crystalline PLLA [12]. Therefore, this work also extends such studies for PLLA crystallized at different temperatures and thus exhibiting distinct lamellar morphologies.

Fig. 1 Scheme of the lamellar morphology considering the three-phase model. The long period as detected by smallangle X-ray scattering will combine the thicknesses of the crystalline layer, $L_{\mathrm{c}}$, the mobile amorphous phase, $L_{\mathrm{ma}}$, and the rigid amorphous phase, $L_{\mathrm{ra}}$. A CRR is schematically represented in the mobile phase, occupying part of the rigid amorphous layer

\section{Experimental}

PLLA of commercial grade (Resomer ${ }^{\circledR}$ L 210) was from Boehringer Ingelheim, with molecular weights of $M_{\mathrm{n}}=$ 180,000 and $M_{\mathrm{w}}=220,000$ and evaluated from gel permeation chromatography (Shimadzu, LC 10A, Japan) using polystyrene as standard and chloroform as solvent. The specific optical rotation measurement of the polymer allowed to estimate a content of L-lactic acid above $99 \%$. PLLA plates were obtained by hot pressing at about $200^{\circ} \mathrm{C}$ and then quenching in cold water (around $15{ }^{\circ} \mathrm{C}$ ). Isothermally cold-crystallized specimens were prepared by annealing the plates for $12 \mathrm{~h}$ in an oven at temperatures ranging between 95 and $165{ }^{\circ} \mathrm{C}$ from the glassy state. After annealing, all the specimens were quenched through air to stop further crystallization. More experimental details related to the preparation of the samples and other complementary tests performed can be found elsewhere [11].

DMA experiments were performed on a DMA7e PerkinElmer analyser with controlled cooling accessory, and highpurity helium was used as a purge gas. Samples with dimensions $0.4 \times 4.4 \times 10 \mathrm{~mm}^{3}$ were cut from the PLLA plates mentioned above and were analysed in the tensile mode. The DMA experiments were performed in isochronal conditions from 20 to $110{ }^{\circ} \mathrm{C}$ at a heating rate of $3{ }^{\circ} \mathrm{C}$ $\min ^{-1}$ and a frequency of $1 \mathrm{~Hz}$. A dynamic stress of amplitude $1 \mathrm{MPa}$ and a static stress of amplitude $1.2 \mathrm{MPa}$ were applied in all the DMA experiments. Before the experiments, all the necessary thermal and mechanical calibrations were performed.

\section{Results and discussion}

DMA allows to obtain information about the viscoelastic properties of materials at distinct temperatures and frequencies. In a DMA experiment, the sample undergoes repeated small amplitude strains in a cyclic manner. Polymeric segments perturbed in this way store a portion of the imparted energy elastically and dissipate a portion in the form of heat. In this case, the modulus acquires a complex form, $E^{*}=E^{\prime}+i E^{\prime \prime}$ $[13,14]$. The quantity $E^{\prime}$, the storage modulus, is a measure of the energy stored elastically, whereas $E^{\prime \prime}$ is the loss modulus that is a measure of the energy lost as heat. The loss factor is defined as $\tan \delta=E^{\prime \prime} / E^{\prime}, \delta$ being the angle between the in-phase and out-of-phase components in the cyclic motion and measures the damping capability of the material. Figure 2 shows the temperature dependence of the storage modulus and the loss factor for the PLLA samples crystallized at different temperatures $\left(T_{\mathrm{c}}\right)$ at a fixed frequency of $1 \mathrm{~Hz}$. The presence of $\alpha$-relaxation is clear in this figure, as revealed by the presence of a well-defined peak in $\tan \delta$ 

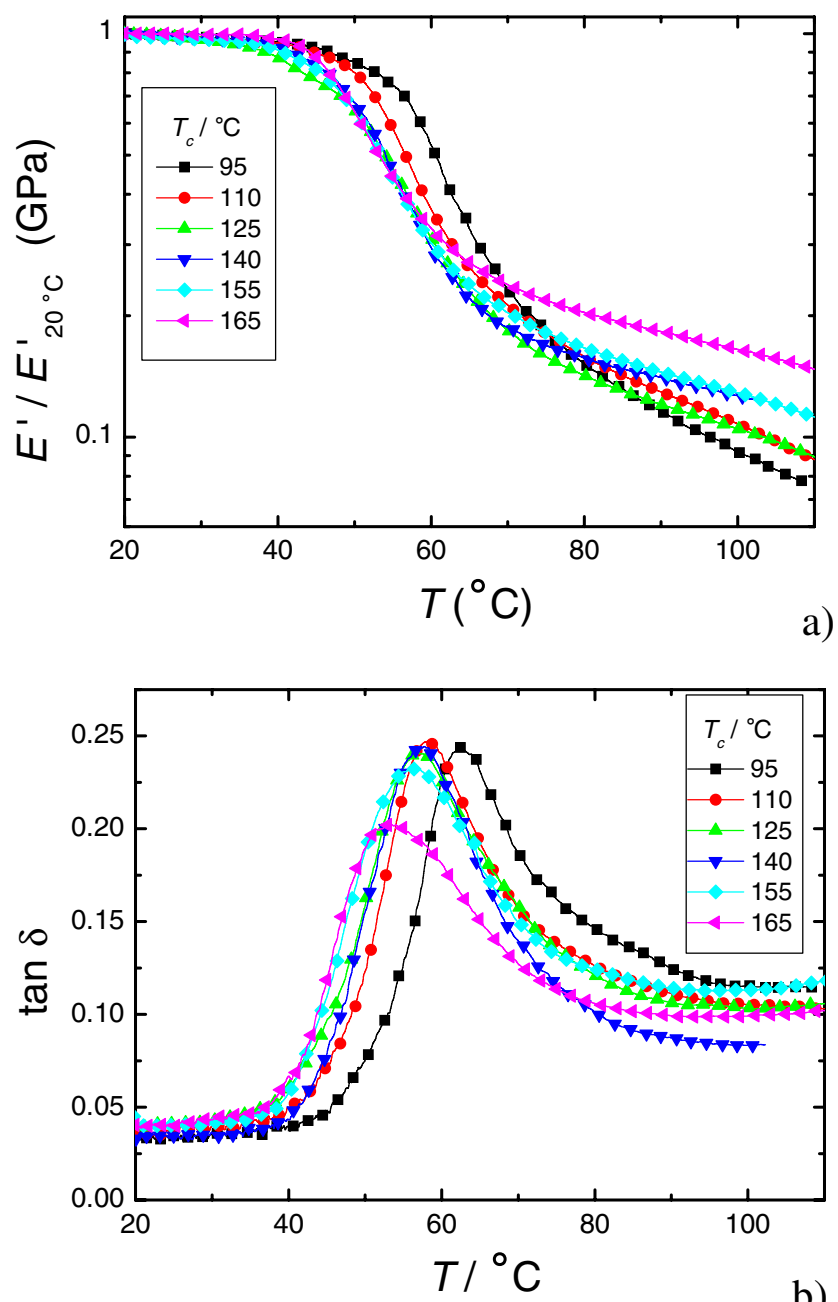

Fig. 2 Storage modulus (a) and loss factor (b) against temperature for PLLA fully crystallized at different crystallization temperatures $\left(T_{\mathrm{c}}\right)$, recorded at $1 \mathrm{~Hz}$ and during a heating scan at $3{ }^{\circ} \mathrm{C} \min ^{-1}$

around $T_{\mathrm{g}}$, accompanied by a strong decrease in $E^{\prime}$, and it is associated to the occurrence of the glass transition upon heating.

The normalized storage modulus in Fig. 2a allows to perceive the strength of the decrease in $E^{\prime}$ during the transition from the glassy to the liquid state of the mobile amorphous fraction within the materials. We reported before that as $T_{\mathrm{c}}$ increases, the overall crystallinity increases, and the corresponding content of the mobile amorphous phase decreases [11]. This explains why the rubbery plateau tends to stabilize at a higher relative modulus as $T_{\mathrm{c}}$ increases because the drop in $E^{\prime}$ will be intimately related to the content of the amorphous counterpart. Another consequence of the decrease in the amorphous content is the tendency for the corresponding decrease in the intensity of the loss factor peak (Fig. 2b).
The loss factor peaks clearly present an ascending behaviour at the low-temperature side, but above $T_{\mathrm{g}}, \tan \delta$ stabilizes to values around 0.1 , rather than descending to values close to the ones found below $T_{\mathrm{g}}$. This behaviour can be detected by DMA in different materials during the occurrence of the glass transition phenomenon, but no conclusive explanation has yet been provided. High damping capability above $T_{\mathrm{g}}$ may affect the properties of the materials and can be relevant for their processing. A physical model was proposed to elucidate the origin of this phenomenon, which included a typical viscoelastic response around a relaxation process coupled in series with a Newtonian dashpot [15]. The concept behind the model suggests that an irreversible flow, modelled by the viscous dashpot, takes place more easily above $T_{\mathrm{g}}$ due to the slippage of polymeric chains. This dissipative process could add some extra contribution in $\tan \delta$. When the main relaxation was described by this modified solid linear standard [15], it was found that the addition of the dashpot leads to an expression of $\tan \delta$ having a positive and additive term relatively to the pure solid linear standard, which could explain qualitatively the existence of a plateau above $T_{\mathrm{g}}$ in the $\tan \delta$ versus $T$ plot.

The most important result in Fig. $2 \mathrm{~b}$ is the consistent shift of the position of the loss factor towards lower temperature as $T_{\mathrm{c}}$ increases. As the samples are fully transformed, as seen by the full spherulitic occupation of the volume from parallel optical microscopy observations [10], this shift in $T_{\mathrm{g}}$ should be related to the different environment felt by the mobile amorphous phase at the lamellar scale. It is well known that the glass transition involves cooperative motions that can progress in a nanometric length scale, and that this process can be interfered by geometrical confinement. Adam and Gibbs [16] introduced the concept of cooperatively rearranging regions (CRR), defined as the smallest region around a relaxing entity that upon a sufficient fluctuation can undergo a transition to a new configurational state independently of its environment. The length scale $\xi$ of the CRRs assigned to the glass transition relaxation in glass formers increases with decreasing temperature, as more extensive cooperativity is needed for the molecular entities to move, diverging near the so-called Vogel temperature. Generally, $\xi$ at $T_{\mathrm{g}}$ ranges between 1.0 and $3.5 \mathrm{~nm}$ [17].

Schick and Donth [18] have proposed that in a semicrystalline system, the inner mobile amorphous phase would be disturbed with respect to the dynamic glass transition of the amorphous bulk phase if the thickness of the mobile amorphous layer is smaller than the typical dimension of the CRR at $T_{\mathrm{g}}$ of the bulk material, i.e., $\sim 2 \xi$. In a simple picture, one may consider that during heating from the glassy state, if the distances of the amorphous domains to the rigid walls are higher than $\xi$, then the 
mobility will be reached at a temperature similar to the $T_{\mathrm{g}}$ of the bulk material. If the distance of the amorphous segments to the wall is smaller than $\xi$, the mobility at that temperature is not possible, as the conformational rearrangement cannot take place due to the hindering of the rigid wall. It is known, and observed experimentally [19], that there is a decrease in dynamic correlations and length scales as the temperature increases above $T_{\mathrm{g}}$. Therefore, only at higher temperatures, for sufficient smaller correlation lengths, the segmental mobility can take place without the perturbation of the confining walls that are linked to the amorphous mobile phase through covalent bonds. In an experimental point of view, this will result in a shift of the glass transition temperature towards higher values. Moreover, within the mobile amorphous layer, one may find mobile segments at different distances from the confining walls, enlarging the temperature range along which the glass transition takes place. This observation is compatible with the broader distribution of characteristic times observed in semi-crystalline systems, with respect to the corresponding amorphous material. For the specific case of PLLA, such comparison was investigated from DSC measurements [5], where the stretched exponent of the Kohlrausch-Williams-Watts model was found to be smaller for semi-crystalline PLLA $(\beta=0.35)$ than for the amorphous counterpart $(\beta=0.40)$. The $\alpha$-relaxation loss peak corresponding to the dynamic glass transition, observed in PLLA by dielectric relaxation spectroscopy, was also found to be described by a broader distribution of relaxation times for the semi-crystalline case [6-7].

From the above considerations, it is not surprising that a correlation between the thickness of the mobile amorphous layer and $T_{\mathrm{g}}$ should exist in semi-crystalline systems (see, for example, [20]). It would also be interesting to understand if the rigid amorphous layer could have any influence on the features of the glass transition dynamics. This possibility has been scarcely analysed in the literature [21-23], and this work intends to further investigate the effect of the rigid amorphous layer using viscoelastic measurements around $T_{\mathrm{g}}$. In a previous work [11], which combined X-ray scattering data and DSC results of PLLA, it was found that with an increasing $T_{\mathrm{c}}$, a consistent increase in the thickness of the rigid amorphous layer could be observed, whereas no conclusive variation in $L_{\text {am }}$ could be found-Fig. 3 resumes some of the data obtained from that work. Therefore, PLLA seems to be an interesting system where one can, within limited possibilities, produce materials having rigid amorphous layers with different thickness while maintaining approximately the thickness of the mobile amorphous layer. At the same time, DSC results revealed a decrease in $T_{\mathrm{g}}$ with an increasing $T_{\mathrm{c}}$ [11]. The same trend between $T_{\mathrm{g}}$ and $T_{\mathrm{c}}$ was also observed by DSC some years ago for PLLA [24]; however, the authors did

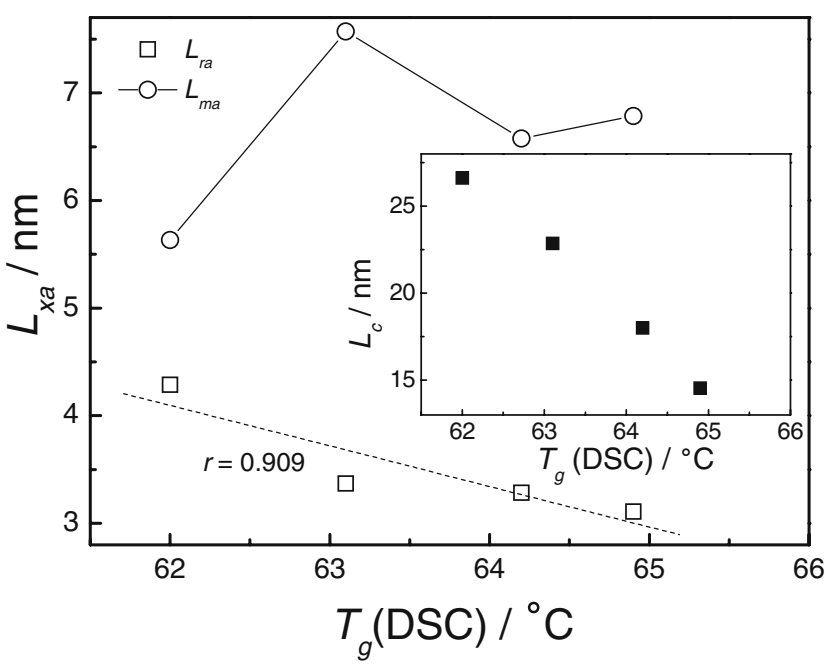

Fig. 3 Plot of $L_{\mathrm{ma}}$ (open circles), $L_{\mathrm{ra}}$ (open squares) and $L_{\mathrm{c}}$ (filled squares in the inset graphics) versus $T_{\mathrm{g}}$ for cold-crystallized PLLA (data taken from [11]). For the $L_{\mathrm{ra}}$ data, a linear regression is presented

not provide any explanation for this behaviour. On the contrary, they referred that the opposite trend should be expected due to the decrease in mobility associated with a higher crystallinity degree as $T_{c}$ increases [24]. In the present work, we can also observe a shift of the $\tan \delta$ peak towards lower temperatures with an increasing $T_{\mathrm{c}}$ (Fig. 2b), compatible with the calorimetric data. Figure 4 shows such variation in more detail, suggesting in a first approximation a linear relationship between the temperature of maximum $\tan \delta, T_{\max }$, and $T_{\mathrm{c}}$.

From viscoelastic measurements, it was then confirmed that when the thickness of the rigid amorphous layer increases, the glass transition shifts to lower temperatures. The rigid amorphous phase should not participate directly in the glass transition; that is, it does not contribute, for example, to the increase in the heat capacity across $T_{\mathrm{g}}$ nor contribute to the $\alpha$-relaxation loss peak in spectroscopic experiments. However, the results obtained for PLLA suggest that this phase could influence, to some extent, the glass transition features. A possible explanation for the observations found in this work could be related to the fact that the micromechanical and dynamic properties of the rigid amorphous phase are quite different from those of the crystalline layer: The lower density and the lack of chain organization make the rigid amorphous phase more ductile than the crystalline one; this may enable the existence of local mobility within the polymeric chains, which could not be observed in the regular structure of the crystalline phase.

Taking into account the typical characteristic length of the CRR [17] and the average thickness of the mobile amorphous layer (Fig. 3), it is expected that the CRR for the chain segments that are in the central region of this layer would not be affected by the rigid amorphous layer. 
Fig. 4 Temperature of maximum loss factor, $T_{\max }$ (squares), and width at half-height for the $\tan \delta$ peak, $W_{1 / 2}$ (circles), as a function of crystallization temperature $\left(T_{\mathrm{c}}\right)$, taken from the data of Fig. 2b. Linear regressions for the two representations are also shown (solid lines). The scheme at the right-hand side explains how $W_{1 / 2}$ was obtained: The peak describing the glass transition in the $\tan \delta$ trace (solid line) is fitted using a simple Gaussian function (dotted line), where $W_{1 / 2}$ is estimated

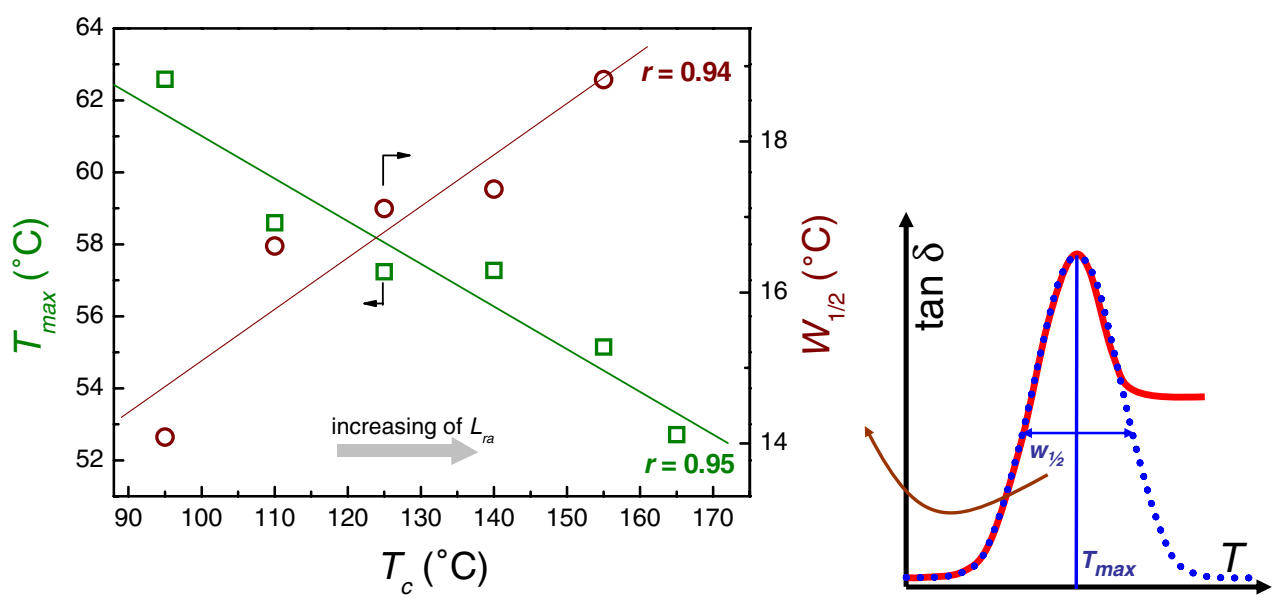

However, if the segments are at a distance lower than $\xi$ from the rigid amorphous phase, then this phase might be included in the CRR, as the scheme of Fig. 1 intends to illustrate. Therefore, upon the cooperative motions of such kind of chain segments within the mobile amorphous layer, the molecular organization of the rigid amorphous layer located within the CRR could be adapted up to a certain extent, in such a way that the segmental motions could be accomplished. The motion of the segments in the centre of the CRR will be possible if some low-extent conformational adjustments inside the rigid amorphous phase permit the occurrence of the overall cooperative conformational motions. Note that if no rigid amorphous layer would exist, or if its thickness is sufficiently small, such motions could not take place, as the CRR of the segments that are at a distance lower than $\xi$ would include a part of the crystalline layer that could not be involved in the dynamic event. In that case, the temperature must increase until the CRR is small enough to exclude the crystalline fraction; that is, an increase in $T_{\mathrm{g}}$ should be observed. Therefore, this possible model permits to expect an increase in $T_{\mathrm{g}}$ with the decrease in the thickness of the rigid amorphous layer. This is, in fact, the trend observed in this work for PLLA.

This model suggests that the rigid amorphous phase possesses some degrees of freedom that could help the segments in the mobile amorphous layer to accomplish their cooperative motions. This "pillow" effect occurring in the rigid amorphous phase is expected to involve low amplitude motions within this phase: We suggest that just small free volume fluctuations or rotational/translational motions of a few monomers could be enough to facilitate the conformational motions occurring in the mobile amorphous phase, especially in the edges of the CRR. Note that the shape of CRRs in glassy liquids are not completely compact: Both computer simulations and theory suggest that the geometry of such regions could be described by string-like or fractal morphology [25]. Therefore, we may hypothesize that motions within some of such irregular regions could take place in the more compact rigid amorphous phase. It must be noticed that the hypothesis proposed in this work is just a possible explanation based on the observed data, which should be confirmed based on further experimental or theoretical information.

It would be expected that the involvement of the rigid amorphous phase in the motions occurring in the mobile phase could diversify the molecular environments felt by the segments during conformational changes, enlarging the distribution of relaxation times. Therefore, besides the change in $T_{\mathrm{g}}$, it would be interesting to address if the different lamellar morphology also changes the distribution of characteristic times assigned to the glass transition dynamics. The complex response of a polymeric system reflects a spectrum of times, each of one characteristic of a determined type of molecular motion that contributes to the overall mechanical response, and that is inversely proportional to the frequency of the corresponding motions. For isothermal experiments, the broadness of the loss peak is higher for broader distributions of characteristic times. For systems with similar temperature dependencies of the characteristic times, such as the different materials analysed in this work, one should also expect that the broadness of the loss peaks in the frequency axis (isothermal experiments) and in the temperature axis (isochronal experiments) should be correlated. This relationship was explained qualitatively, for example, by Cameron et al. [26]. Therefore, in this work, it was used as a qualitative approach to have an indication about the broadness of the distribution of characteristic times by measuring the broadness of the loss factor peak obtained by DMA. This procedure was already used by other authors [21]. The isochronal $\tan \delta$ data were fitted by a simple function, and we assume a correlation between the broadness of the distribution of characteristic times and the half-height width of the peak $W_{1 / 2}$ (see right-hand-side scheme in Fig. 4). Figure 4 shows $W_{1 / 2}$ as a function of the crystallization temperature, where a relationship seems to exist. However, 
the statistical significance of this finding should be more deeply confirmed by also using other polymeric systems. According to the previous results, we may then suggest that as the thickness of the rigid amorphous phase decreases, $T_{\mathrm{g}}$ increases, and we concomitantly observe an increase in the broadness of the time spectrum. In a future work, broadband dielectric relaxation experiments in such system will be reported, where more information on the trend in the distribution of relaxation times of the lamellar morphology will be discussed.

\section{Conclusions}

This work showed that DMA could be a suitable technique to relate the crystalline morphology of semi-crystalline polymers and the corresponding glass transition features. For the specific case of PLLA, a decrease in the glass transition temperature with an increasing crystallization temperature was observed. This finding was consistent with previously reported DSC results [11]. The decrease in $T_{\mathrm{g}}$ was correlated with the changes in the thickness of the rigid amorphous phase. It was suggested that this phase, although not participating directly in the cooperative segmental motions, could have some translational/rotational degrees of freedom. Such local mobility could allow for the accommodation of the segmental mobility of chains in the mobile amorphous phase, in which the edges of the CRR overlap with the rigid amorphous phase. For thinner rigid amorphous layers, this process could be more hindered as the mobile amorphous phase would be in a more direct contact with the rigid crystalline layer. In that case, such cooperative segmental motions could only take place at higher temperatures, where the characteristic lengths of the cooperative rearranging regions are smaller. Besides the changes in $T_{\mathrm{g}}$, the increase in the crystallization temperature broadens the loss factor peak, which could indicate an increase in the broadness of the distribution of relaxation times. Therefore, the results reported in this study provided further data on the dependence of the glass transition on the geometrical confinement provoked by the crystalline lamellae in semi-crystalline polymers, and also supply information about the general viscoelastic behaviour of PLLA crystallized at different conditions and thus exhibiting different morphologies.

Acknowledgment The authors acknowledge the financial support provided by FCT, through the POCTI and FEDER programmes, and POCTI/FIS/61621/2004. Y. Wang also thanks the PostDoc grant from Portuguese FCT (SFRH/11497/2002).

\section{References}

1. Langer R, Vacanti JP (1993) Science 260:920

2. Ikada Y, Tsuji H (2000) Macromol Rapid Commun 21:117

3. Kim HD, Bae EH, Kwon IC, Pal RR, Nam JD, Lee DS (2004) Biomaterials 25:2319

4. Wang Y, Mano JF (2006) J Appl Polym Sci 100:2628

5. Mano JF, Gómez Ribelles JL, Alves NM, Salmerón Sanchez M (2005) Polymer 46:8258

6. Dionísio M, Viciosa MT, Wang Y, Mano JF (2005) Macromol Rapid Commun 26:1423

7. Brás AR, Viciosa MT, Wang Y, Dionisio M, Mano JF (2006) Macromolecules 39:6513

8. Salmerón Sánchez M, Gómez Ribelles JL, Hernández Sánchez F, Mano JF (2005) Thermochim Acta 430:201

9. Wang Y, Gómez Ribelles JL, Salmerón Sánchez M, Mano JF (2005) Macromolecules 38:4712

10. Wunderlich B (2005) Macromol Rapid Commun 26:1521

11. Wang Y, Funari SS, Mano JF (2006) Macromol Chem Phys 207:1262

12. Mano JF (2005) Macromol Biosci 5:337

13. McCrum NG, Read BE, Williams G (1991) Anelastic and dielectric effects in polymer solids. Dover, New York

14. Ferry JD (1980) Viscoelastic properties of polymers, 3rd edn. Wiley, New York

15. Alves NM, Lanceros-Méndez S, Mano JF, Gómez-Ribelles JL (2002) Defect Diffus Forum 206(2):131

16. Adam G, Gibbs JH (1965) J Chem Phys 43:139

17. Hempel E, Hempel G, Hensel A, Schick C, Donth E (2000) J Phys Chem B104:2460

18. Schick C, Donth E (1991) Physica Scripta 43:423

19. Berthier L, Biroli G, Bouchaud J-P, Cipelletti L, El Marsi D, l'Hôte D, Ladieu F, Pierno M (2005) Science 310:1797

20. Dobbertin J, Hensel A, Schick C (1996) J Therm Anal 47:1027

21. Hong P-D, Chuang W-T, Yeh W-J, Lin T-L (2002) Polymer 43:6879

22. Natesan B, Xu H, Ince BS, Cebe P (2004) J Polym Sci B: Polym Phys 42:777

23. El-Taweel SH, Höhne GWH, Mansour AA, Stoll B, Seliger H (2004) Polymer 45:983

24. Iannace S, Nicolais L (1997) J Appl Polym Sci 64:911

25. Stevenson JD, Schmalian J, Wolynes PG (2006) Nat Phys 2:268

26. Cameron N, Cowie JMG, Ferguson R, Gómez Ribelles JL, Más Estelles J (2002) Eur Polym J 38:597 